\title{
Detection of Growth Hormone Deficiency in Adults with Chronic Traumatic Brain Injury
}

\author{
Lisa A. Kreber,,3 Grace S. Griesbach,, ${ }^{1,3}$ and Mark J. Ashley ${ }^{1,3}$
}

\begin{abstract}
This study examined the prevalence of growth hormone deficiency (GHD) in patients with traumatic brain injury (TBI) during the post-acute phase of recovery and whether GHD was associated with increased disability, decreased independence, and depression. A secondary objective was to determine the accuracy of insulin-like growth factor-1 (IGF-1) levels in predicting GHD in patients with TBI. Anterior pituitary function was assessed in 235 adult patients with TBI through evaluation of fasting morning hormone levels. GH levels were assessed through provocative testing, specifically the glucagon stimulation test. GHD was diagnosed in a significant number of patients, with $45 \%$ falling into the severe GHD $(\leq 3 \mu \mathrm{g} / \mathrm{L})$ category. IGF-1 levels were not predictive of GHD. Patients with GHD were more disabled and less independent compared with those patients who were not GHD. Those patients with more severe GHD also showed decreased levels of cortisol and testosterone. Symptoms of depression were also more prevalent in this group. In addition, patients with severe GHD had delayed admission to post-acute rehabilitation. This study confirms the high prevalence of GHD in patients with TBI and the necessity to monitor clinical symptoms and perform provocative testing to definitively diagnose GHD.
\end{abstract}

Key words: adult brain injury; clinical management of CNS injury; growth hormone deficiency; hypopituitarism; human studies

\section{Introduction}

$\mathbf{T}$ Raumatic Brain InJURy (TBI) is a leading cause of death and disability in the United States. ${ }^{1}$ Life-long consequences of sustaining a TBI can include impairments in physical, cognitive, and psychosocial functioning. ${ }^{2}$ It is estimated that 5.3 million people in the United States are currently living with disabilities secondary to TBI. ${ }^{3}$

Hypopituitarism is a frequent, yet undertreated consequence of TBI that may contribute to morbidity and poor recovery. Reports of the prevalence of anterior hypopituitarism in the chronic phase of TBI varies, ranging from $15-50 \%{ }^{4-12}$ Growth hormone deficiency (GHD) is the most common anterior pituitary deficit after TBI, with prevalence ranging from $6-33 \%$ in the chronic phase of recovery, ${ }^{4-14}$ depending on such factors as TBI chronicity, injury severity, age of onset, and the methods used to diagnose pituitary hormone dysfunction.

Despite clear evidence that a large number of persons with TBI have GHD, few patients are actually screened for GHD after TBI. Symptoms reported by persons with adult onset GHD significantly overlap with deficits reported after TBI and may result in a missed diagnosis of GHD and delayed treatment of GHD in persons with TBI. Memory and concentration impairments, decreased quality of life, anxiety, depression, social isolation, hyperlipidemia, weight gain, osteoporosis, and exercise intolerance are routinely reported by both persons with adult onset GHD without TBI and those with TBI alone. ${ }^{14,15}$ Therefore, proper screening and evaluation of GH function is essential to diagnose GHD after TBI.

$\mathrm{GH}$ is a pleiotropic hormone synthesized from the somatotroph cells in the anterior pituitary gland and released, in pulsatile bursts, into the bloodstream to stimulate the production and release of insulin-like growth factor-1 (IGF-1). GH has been shown to stimulate proliferation and differentiation of cells; influence migration and survival of neural cells; stimulate the genesis of neurons, astrocytes, endothelial cells, and oligodendrocytes; and to promote myelination and neuronal arborization. ${ }^{16,17}$ IGF-1 is the major GH-dependent peptide ${ }^{18}$ and is used as a surrogate biomarker for GH. ${ }^{15,19,20}$ IGF-1 exerts many of the same effects on the brain during development and in adulthood as $\mathrm{GH}^{17,21}$

Reliance on IGF-1 as an assessment of GH function after TBI is standard practice, but its use needs to be re-evaluated because $50 \%$ of adults with GHD have IGF-1 levels within the normal reference range. ${ }^{22}$ Similarly, patients with a normal GH response can have low IGF-1 levels. Direct serum assessment is unreliable because of the pulsatile release of $\mathrm{GH}$ and results in serum fluctuations within a

\footnotetext{
${ }^{1}$ Centre for Neuro Skills and ${ }^{2}$ Department of Neurosurgery, ${ }^{3}$ Centre for Neuro Skills - Clinical Education and Research Foundation, David Geffen School of Medicine at UCLA, Bakersfield, California.

(c) Lisa A. Kreber, et al., 2016; Published by Mary Ann Liebert, Inc. This Open Access article is distributed under the terms of the Creative Commons Attribution Noncommercial License (http://creativecommons.org/licenses/by-nc/4.0/) which permits any noncommercial use, distribution, and reproduction in any medium, provided the original author(s) and the source are credited.
} 
24-h period. ${ }^{23}$ Thus, provocative testing is essential to definitively diagnose GHD. ${ }^{15,20}$

Peak GH secretion during provocative testing is used to assess the capacity of the pituitary to release $\mathrm{GH}^{24}$ The insulin tolerance test (ITT) is considered the "gold standard" in provocative tests for diagnosing GHD ${ }^{15,25,26}$; however, it cannot be safely performed in patients with seizures or severe cardiovascular disease. ${ }^{27,28}$ This contraindication limits its use in patients with TBI. The glucagon stimulation test (GST) has comparable diagnostic accuracy and reliability as the $\operatorname{ITT}^{20,29,30}$ and is well tolerated in patients with TBI. A single provocative test is sufficient for the diagnosis of GHD in adults. ${ }^{31}$

It was hypothesized that a large percentage of patients with TBI would exhibit GHD, as measured by GST provocative testing. Exploratory analyses also examined whether GHD was associated with increased disability and depression, because these symptoms are present in patients with TBI and in patients with isolated GHD. In addition, it was hypothesized that IGF-1 would not be a sensitive diagnostic marker of GHD.

\section{Methods}

All procedures were reviewed and approved by the Centre for Neuro Skills' Institutional Review Board.

\section{Eligibility}

Adult patients with a diagnosis of TBI admitted to the Centre for Neuro Skills, a brain injury residential transitional rehabilitation facility, were assessed for neuroendocrine dysfunction via the endocrine laboratory panel described below. Patients were excluded from the study if they had a history of documented endocrine abnormalities, contraindications to GH replacement (i.e., a history of cancer or a brain tumor), and psychiatric and/or behavioral diagnoses that prevented completion of the study. A total of 235 patients were included for analysis in this study. Most patients were in the moderate $(80 \%)$ level of disability according to the Disability Rating Scale (DRS) on admission. Table 1 contains demographic data for patients included in the study.

\section{Hormone assays}

After overnight fasting, serum levels of thyroid stimulating hormone $(\mathrm{TSH})$, triiodothyronine $\left(\mathrm{T}_{3}\right)$, thyroxine $\left(\mathrm{T}_{4}\right)$, follicle stimulating hormone $(\mathrm{FSH})$, luteinizing hormone $(\mathrm{LH})$, total and free testosterone (males only), estradiol (females only), prolactin, cortisol (a.m.) and IGF-1 were examined ( $\sim 0600$ h). Hormone assays

Table 1. Demographics

\begin{tabular}{lc}
\hline Demographics & Mean \pm SEM \\
\hline Age at injury (years) & $42 \pm 0.95$ \\
Injury chronicity (days) & $367 \pm 46.92$ \\
Male sex (number (\%)) & $198(84 \%)$ \\
\hline Mechanism of injury & Number (\%) \\
\hline Motor vehicle accident & $77(33 \%)$ \\
Motor-pedestrian accident & $9(4 \%)$ \\
Motor-bicycle accident & $4(2 \%)$ \\
Motorcycle accident & $24(10 \%)$ \\
Assault & $17(12 \%)$ \\
Fall & $73(31 \%)$ \\
Blow to head & $17(8 \%)$ \\
\hline
\end{tabular}

SEM, standard error of the mean. were completed within a week of admission to the rehabilitation facility. GST and serum IGF-1 levels were assessed on all patients who qualified for inclusion.

The GST was performed after an initial baseline blood draw. Glucagon, $1 \mathrm{mg}$, was administered intramuscularly, and blood draws occurred at 0 (baseline), 90, 120, 150, and $180 \mathrm{~min}$ for GH. Blood was centrifuged, and serum was frozen with dry ice until analysis by an independent laboratory (Esoterix, Inc., Calabasas Hills, CA).

\section{Functional outcomes}

All patients were assessed on a variety of functional outcome measures within 2 weeks of the hormone assays. These functional outcomes included the DRS, ${ }^{32}$ Independent Living Scale ${ }^{33}$ (ILS), Mayo-Portland Adaptability Inventory- $4^{34}$ (MPAI-4), Community Integration Questionnaire ${ }^{35}$ (CIQ), Occupational Status Scale Sc $^{36}$ (OSS), and the Living Status Scale ${ }^{36}$ (LSS). These outcome scales are indicative of disability (DRS); competence and assistance needed for activities of daily living, initiation, and behavior (ILS); ability, adjustment, and participation (MPAI-4); community integration (CIQ); occupational or avocational status (OSS); and level of supervision (LSS) in the post-acute phase of brain injury. The Beck Depression Inventory-II ${ }^{37}$ (BDI-II) was administered to patients to assess current symptoms of depression.

\section{Data analysis}

Samples for TSH, $\mathrm{T}_{3}, \mathrm{~T}_{4}, \mathrm{FSH}, \mathrm{LH}$, total and free testosterone, estradiol, prolactin, cortisol (a.m.), and IGF-1 were analyzed by an independent commercial laboratory (Esoterix, Inc.) for analysis of anterior pituitary function. Standard reference ranges were used to determine whether hormone levels were out of the normal range.

For IGF-1, laboratory reference ranges were based on age and sex. This reference range was used to categorize patients as either "IGF-1 Deficient" or "IGF-1 Normal." GH levels were analyzed using an immunochromatographic membrane assay. Patients with a peak $\mathrm{GH}$ of $\leq 3.0 \mu \mathrm{g} / \mathrm{L}$ were considered to be severely GHD (sGHD), and patients with a peak GH of $3.1-5.0 \mu \mathrm{g} / \mathrm{L}$ were considered moderately GHD (mGHD). Patients with a peak GH of $\geq 5.1 \mu \mathrm{g} / \mathrm{L}$ were considered not GHD (nGHD).

All statistical analyses were performed using Statistical Package for Social Sciences (SPSS, Inc., Chicago, IL) version 22. Data are reported as mean \pm standard error of the mean (SEM). Raw data for demographic variables, hormone assays, and functional outcome measures were compared through one-way analysis of variance (ANOVA), and when appropriate, Bonferroni post hoc tests were performed. All comparisons were Bonferroni corrected. The level of significance was chosen as $p<0.05$.

\section{Results}

\section{GH deficiency}

Results from the GST indicated that 181 (77\%) of the 235 patients with TBI tested for GHD had some level of GHD, ranging from sGHD to mGHD (Table 2).

A significant difference existed between the sGHD and both the mGHD and $n G H D$ groups $(\mathrm{F}(2,234)=6.96 ; p=0.001)$ in latency to be admitted to post-acute rehabilitation (Fig. 1). The sGHD group took longer to be admitted for rehabilitation compared with the other groups. Comparisons between patients with sGHD, mGHD, or nGHD revealed no significant differences in age at TBI onset, age at the time of admission to rehabilitation, or sex.

\section{IGF-1 sensitivity and accuracy}

A Pearson correlation analysis revealed no significant correlation between peak GH levels and IGF-1 levels $(r=0.064, n=235)$. 
Table 2. Patients with a Diagnosis of Growth Hormone Deficiency via the Glucagon Stimulation Test

\begin{tabular}{lc}
$\begin{array}{l}\text { GH category (mean } \pm \text { SEM) } \\
\text { Cutoff for the category }\end{array}$ & $\begin{array}{c}\text { Percentage } \\
(\mathrm{N})\end{array}$ \\
\hline sGHD $(1.38 \pm 0.09)$ & $45 \%$ \\
Peak GH $\leq 3 \mu \mathrm{g} / \mathrm{L}$ & $(\mathrm{N}=105)$ \\
mGHD $(4.54 \pm 0.09)$ & $17 \%$ \\
Peak GH $3.1-5.0 \mu \mathrm{g} / \mathrm{L}$ & $(\mathrm{N}=41)$ \\
nGHD $(10.97 \pm 0.60):$ & $38 \%$ \\
Peak $\mathrm{GH} \geq 5.1 \mu \mathrm{g} / \mathrm{L}$ & $(\mathrm{N}=89)$ \\
\hline
\end{tabular}

The majority of patients had a diagnosis of severe growth hormone deficiency (sGHD).

$\mathrm{GH}$, growth hormone; SEM, standard error of the mean; mGHD. moderate growth hormone deficiency; nGHD, no growth hormone deficiency.

To address the accuracy and sensitivity of IGF-1 reference ranges in screening patients with TBI for GHD, IGF-1 levels were compared with GST results. According to standard reference ranges, based on age and sex, there were no patients with abnormally high levels of IGF-1. Patients were then further classified based on the results of the GST, as either GHD or nGHD based on the results of the GST. To be included in the GHD group, a patient needed to have a peak GH level of $\leq 5 \mu \mathrm{g} / \mathrm{L}$, which would include the sGHD and mGHD categories in this study. Forty-two percent of patients with TBI whose IGF-1 level fell within the normal reference range for their age and sex were GHD, indicating that if IGF-1 levels were relied on in isolation, a false negative result would have been obtained (Table 3).

\section{Other hormone assays}

There was a significant difference in total testosterone $(\mathrm{F}(3,179)$ $=4.147 ; p=0.007$ ) between the sGHD group and the normal GH group (Fig. 2a). Results also indicated a significant difference in morning cortisol level $(\mathrm{F}(3,209)=3.365 ; p=0.020)$ between the sGHD and the mGHD groups (Fig. 2b). No significant differences in admission values of TSH, FSH, LH, estradiol, or prolactin were observed. IGF-1 levels approximated significance $(p=0.099)$ between the GH groups.

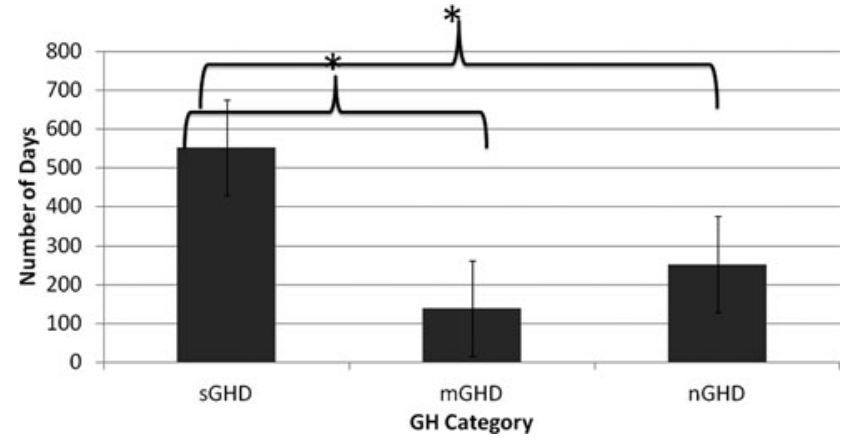

FIG. 1. Comparison of injury chronicity by growth hormone (GH) category. The severe growth hormone deficiency (sGHD) group took significantly longer to be admitted to post-acute rehabilitation than the moderate growth hormone deficiency (mGHD) group and the no GHD group (nGHD). The mGHD group and the nGHD group were not significantly different from each other. Each value represents the mean \pm standard error of the mean, $* p<0.05$.
Table 3. Sensitivity of Insulin-Like Growth Factor-1 Values in the Diagnosis of Growth Hormone Deficiency

\begin{tabular}{ccc}
\hline & IGF-1 Deficient & IGF-1 Normal \\
\hline GHD & $20 \%$ & $42 \%$ \\
peak $\mathrm{GH} \leq 5 \mu \mathrm{g} / \mathrm{L}$ & $(\mathrm{N}=48)$ & $(\mathrm{N}=98)$ \\
& True & False \\
& Positive & Negative \\
nGHD & $15 \%$ & $23 \%$ \\
& $(\mathrm{~N}=36)$ & $(\mathrm{N}=53)$ \\
& False & True \\
& Positive & Negative \\
& $\mathrm{N}=235$ \\
Reference range for IGF-1 based on age and gender &
\end{tabular}

Patients were categorized into insulin-like growth factor-1 (IGF-1) deficient or IGF-1 normal groups based on age and sex reference ranges. Patients were also classified as being growth hormone deficient (GHD) or non-GHD (nGHD) on the basis of results from the glucagon stimulation test (GST).

\section{Functional outcome measures}

No significant differences were observed between the sGHD and the mGHD group on any of the functional outcome measures. Therefore, these were combined into one group, GHD, for all analyses of functional outcome measures. Comparisons were made between the GHD group and the no GHD group (nGHD) for all analyses of functional outcome measures.

DRS. Results indicated a significant difference between the GHD and the nGHD on the DRS at admission $(F(1,205)=6.280$, $p=0.013)$. Patients with GHD were more disabled at admission than patients who had no deficiency (Fig. 3a).

ILS. Results indicated a significant difference between the GHD and the nGHD groups on the ILS at admission ( $F(1$, $200)=4.247, p=0.041$ ). Patients in the GHD group were less able to perform activities of daily living and initiate tasks compared with patients in the nGHD group (Fig. 3b). No significant differences were observed between the GHD and the nGHD groups for the other functional outcome measures (MPAI-4, CIQ, and LSS) at the time of the GST. The GHD group tended to perform worse on the OSS compared with the nGHD $(p=0.055)$.

BDI-II. Results indicated a significant main effect between groups on the BDI-II $(\mathrm{F},(2,101)=4.15, p=0.019$, Fig. 4). Although symptoms of depression were higher in the sGHD, compared with other groups, significance was approached $(p=0.056)$ but not obtained.

\section{Discussion}

\section{GH deficiency is present in the chronic phase of TBI}

Seventy-seven percent of patients in this study had a diagnosis of some level of GHD. Consensus guidelines for TBI conceptualize a continuum of GHD depending on peak GH levels after provocative testing. ${ }^{15,20}$ This continuum was demonstrated in the current study. The majority of the patients in this study fell within the sGHD category (peak GH $\leq 3 \mu \mathrm{g} / \mathrm{L}$ ), based on results of the GST. Prevalence rates for GHD range from $6-33 \%$ in the chronic phase of recovery. ${ }^{4-14}$ The higher prevalence of GHD in this study may be because of the use of the GST to diagnose GHD and the inclusion of patients who had mGHD. 

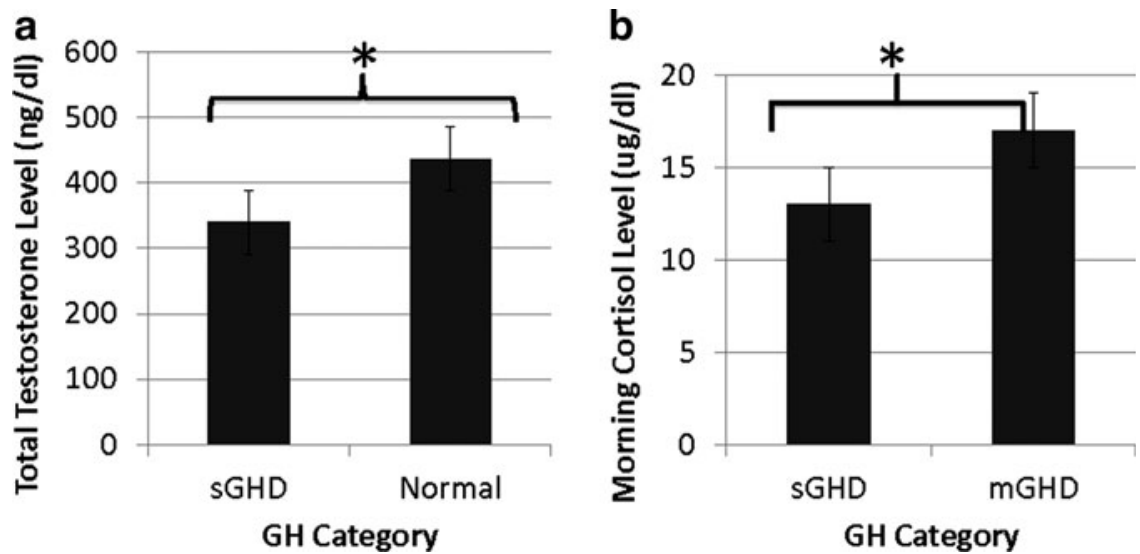

FIG. 2. (a) Comparison of total testosterone levels between the severe growth hormone deficient (sGHD) and the normal growth hormone groups. The sGHD group has significantly lower testosterone levels than the normal growth hormone group. (b) Comparison of morning cortisol levels between the sGHD and the moderate growth hormone deficient (mGHD) groups. The sGHD group has significantly lower cortisol levels than the mGHD group. Each value represents the mean \pm standard error of the mean, ${ }^{*} p<0.05$.

It should be noted that patients in this study were approximately 1 year post-TBI and, therefore, past the point at which pituitary dysfunction would be considered transient. ${ }^{38}$ In fact, patients with sGHD took longer to be admitted to post-acute rehabilitation (Fig. 1), providing further support that pituitary function was stable at the time of hormone evaluation. Although a cause-effect relationship cannot be determined, it may also be that patients with sGHD had more severe brain injuries that resulted in longer hospital and acute rehabilitation lengths of stay. It has been shown that chronic hypopituitarism is associated with more severe brain injuries and increased disability. ${ }^{39}$

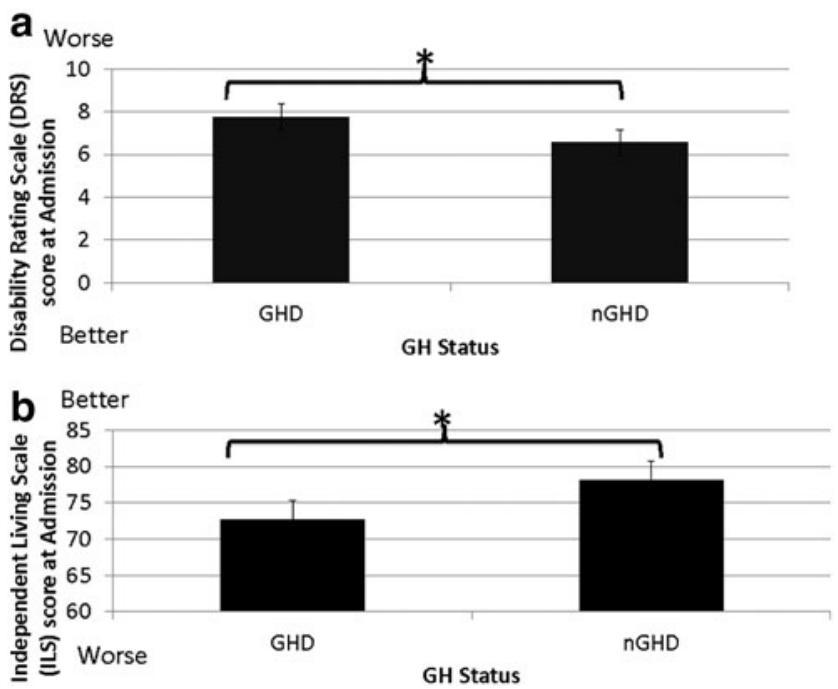

FIG. 3. (a) Comparison of disability levels between the growth hormone deficiency (GHD) groups and the no growth hormone deficiency (nGHD) groups. The GHD group is made up of the severe and moderate GHD groups. The GHD group had higher scores on the Disability Rating Scale indicating higher levels of disability compared with the nGHD group. (b) Comparison of independence levels between the GHD and the nGHD groups. The GHD group had lower scores on the Independent Living Scale (ILS), indicating lower levels of independence compared with the nGHD group. Each value represents the mean \pm standard error of the mean, $* p<0.05$.

\section{IGF-1 levels are not a good predictor of GHD}

This study provides further support that IGF-1 levels are not a good predictor of GHD. It has been reported that approximately $50 \%$ of patients with IGF-1 levels falling within the normal reference range are GHD. ${ }^{22}$ In this study, $42 \%$ of patients whose IGF- 1 level was considered normal for their age and sex were confirmed to be GHD via the GST, with a cutoff of peak GH $\leq 5 \mu \mathrm{g} / \mathrm{L}$. If GHD were diagnosed solely on IGF-1 levels, a notable number of patients would have been missed and withheld from potential treatment (Table 3). Irrespective of the published consensus guidelines that exist for the diagnosis of GHD after TBI, ${ }^{15,20}$ endocrinologists and other healthcare professionals often rely solely on IGF-1 levels to determine GHD, rather than evaluating GH with provocative testing. The results of this study support provocative testing for the diagnosis of GHD in patients with TBI.

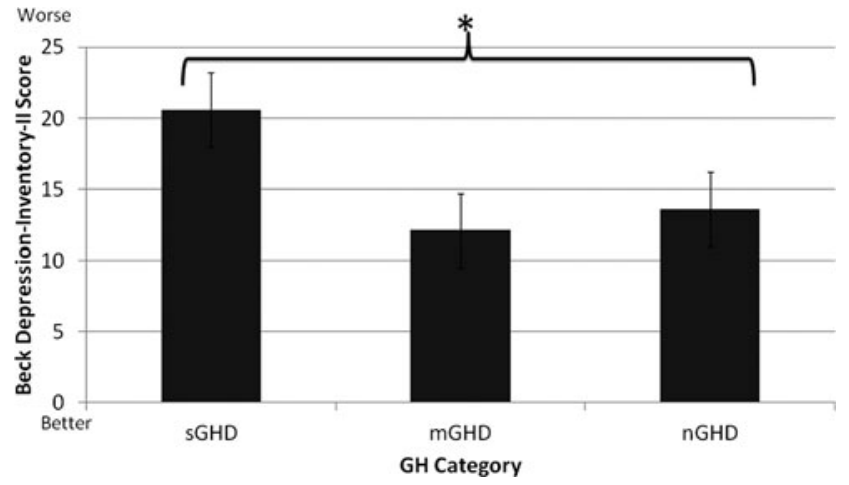

FIG. 4. Comparison of symptoms of depression between the severe growth hormone deficiency (sGHD), moderate growth hormone deficiency (mGHD), and no growth hormone deficiency (nGHD) groups. There was a significant main effect between groups. Although symptoms of depression were higher in the sGHD group, compared with the other groups, significance was approached $(p=0.056)$ but not obtained. Each value represents the mean \pm standard error of the mean, $* p<0.05$. 


\section{Patients with GHD are more likely to have low levels of other hormones}

Patients in this study who presented with sGHD had significantly lower levels of testosterone than nGHD patients (Fig. 2a). Low total testosterone levels have been associated with lower total Functional Independence Measure (FIM) scores at both admission and discharge in the acute rehabilitation setting. ${ }^{40,41}$ These authors suggest that lower levels of total testosterone after TBI may limit the rate or extent of recovery. Likewise, results of the cortisol screening revealed significantly lower levels of morning cortisol in patients with sGHD compared with patients with mGHD (Fig. 2b). A limitation of the current study is that cortisol provocative testing was not undertaken to get an assessment of adrenocorticotrophic (ACTH) functioning. Analogous to IGF-1 not being predictive of GH deficiency, cortisol levels not obtained from provocative testing are not predictive of ACTH deficiency. ${ }^{42}$

\section{Functional outcomes}

Hormone deficiencies can negatively influence recovery from brain injury, even if the patient is undergoing intense rehabilitation. ${ }^{38,43}$ Some evidence exists for greater cognitive dysfunction associated with GHD after TBI. ${ }^{44}$ Results of the current study support these findings in that higher disability scores on the DRS were associated with GHD (Fig. 3a), as were lower levels of independence on the ILS (Fig. 3b). Patients with GHD had more disability than patients who were nGHD. Although not investigated in this study, increasingly evidence suggests that these patients may not achieve the same level of recovery as patients with no GHD.

In a study investigating hypothalamic-pituitary dysfunction after TBI in an acute rehabilitation setting, hormone hypogonadism or low levels of testosterone were observed in $66 \%$ of patients. ${ }^{23}$ The functional implications of hypogonadism were assessed using FIM scores. Patients with low levels of testosterone and IGF-1 had significantly lower FIM change per day scores compared with patients with normal levels of testosterone and IGF-1. ${ }^{23}$

Consistent with these findings, motor and cognitive improvements associated with GH replacement in TBI have been reported. ${ }^{45}$ Undiagnosed hormone deficiencies have the potential to impede rehabilitation and recovery, thus warranting screening for anterior pituitary dysfunction, especially in the chronic phase of recovery. ${ }^{38}$ It is likely that testosterone deficiency contributed to worse functional outcome in these patients. It will be important to further study the contribution of testosterone deficiency to functional outcome in future studies.

\section{Affective disorders}

Patients with SGHD tended to have higher levels of depressive symptoms on the BDI-II (Fig. 4) that may negatively impact recovery from TBI and quality of life. Psychosocial and emotional changes after TBI are common and could be caused not only by the brain injury itself but also by secondary hypopituitarism after TBI. GHD has been associated with increased disability, poor quality of life, and a greater likelihood of depression. ${ }^{39,46}$ Patients with isolated GHD demonstrate a greater rate of depression than those without GHD or GH insufficiency. ${ }^{39}$

As indicated above, patients with sGHD also tended to have lower levels of testosterone compared with the nGHD group. In non-TBI populations, testosterone deficiency has been correlated with depressed mood. ${ }^{47,48}$ This study demonstrates that low levels of testosterone and GHD after TBI may contribute to increased depressive symptoms. Many of the patients in the sGHD group were also testosterone deficient; thus, testosterone deficiency could be contributing to both cognitive and affective symptoms after TBI. Future studies are needed to disentangle the effects of GHD from the effects of testosterone deficiency on symptomatology after TBI.

\section{Physiological considerations}

Burgeoning interest concerning the potential contribution to the pathogenesis of a number of neurodegenerative conditions by TBI points to physiological changes within the central nervous system (CNS) after injury. Neuroendocrine function may well contribute to both the pathogenesis and management of disrupted CNS physiology. Clinical biomarkers for demonstration of cellular proliferation, migration, and survival are not available nor are they for myelination and myelin repair, metabolic stress, or neuronal arborization, all of which may be positively impacted by GH replacement in the injured brain.

The contribution of GH replacement may be found in health issues relating to cardiovascular health (hyperlipidemia and metabolic syndrome), exercise intolerance, osteoporosis, and neuroanatomic and neurophysiologic function as brain injury becomes viewed increasingly as a chronic disease. ${ }^{49}$ IGF-1 production is related to GH availability, among other factors. IGF-1 is an important anti-inflammatory cytokine and plays numerous roles in microglial, astrocytic, endothelial, and neuroinflammatory functions within the CNS.

\section{Limitations and future directions}

There are methodological limitations to the current retrospective analysis that may limit the generalizability of the findings. Because this was a retrospective analysis, there is no control group to compare prevalence of GHD with other populations, which would have minimized the potential for bias and allowed us to rule out other factors, such as injury severity that may have contributed to our findings. In addition, peak cortisol levels were not measured during the GST and would have provided more accurate information regarding cortisol levels in our patient population.

Hormone levels were only obtained at one time point. This did not allow for measuring recovery of pituitary function across time. It is highly unlikely, however, that pituitary function would have resolved across time unless hormone replacement had been initiated. As stated previously, most transient hormone deficiencies resolve by 6 months post-TBI, and these patients were 1 year postTBI. ${ }^{38}$ Functional outcome measures were measured within a few days of blood sampling. It is likely that patients' scores on functional outcome assessments would improve across time, because all patients were engaged in intense rehabilitation. It is unknown, however, whether GHD hindered recovery. Other studies have suggested that although overall recovery occurs, the trajectory of recovery is affected by hormonal deficiencies. ${ }^{40,41}$ In other words, hormone deficiencies may not impede recovery, but may influence the rate of recovery.

\section{Conclusion}

Our study has demonstrated that pituitary dysfunction is still present in a large percentage of patients in the chronic phase of recovery. Reliance on IGF-1 levels in isolation did not accurately predict GHD in the majority of patients with a diagnosis of GHD via provocative testing. Patients with GHD had lower levels of testosterone and cortisol than patients with less severe GHD or nGHD. Significantly increased levels of disability and dependence 
on others for activities of daily living as well as increased symptoms of depression were also more likely to be present in patients with GHD. Further studies are warranted to determine the effect of GHD on the rate of functional recovery from TBI across time.

\section{Author Disclosure Statement}

No competing financial interests exist.

\section{References}

1. Faul, M., Xu, L., Wald, M.M., and Coronado, V.G. (2010). Traumatic brain injury in the United States: Emergency department visits, hospitalizations, and deaths. Centers for Disease Control and Prevention, National Center for Injury Prevention and Control: Atlanta, GA.

2. Dams-O'Connor, K., Spielman, L., Singh, A., Gordon, W.A., Lingsma, H.F., Maas, A. I., Manley, G.T., Mukherjee, P., Okonkwo, D.O., Puccio, A.M., Schnyer, D.M., Valadka, A.B., Yue, J.K., Yuh, E.L.; TRACK-TBI Investigators. (2013). The impact of previous traumatic brain injury on health and functioning: A TRACK-TBI study. J. Neurotrauma 30, 2014-2020.

3. Selassie, A.W., Zaloshnj, E.P., Langlois, J.A., Miller, T., Jones, P., and Steiner, C. (2008). Incidence of long-term disability following traumatic brain injury hospitalization, United States, 2003. J. Head Trauma Rehabil. 23, 123-131.

4. Bondanelli, M., De Marinis, L., Ambrosio, M.R., Monesi, M., Valle, D., Zatelli, M.C., Fusco, A., Bianchi, A., Farneti, M., and degli Umberti, E.C. (2004). Occurrence of pituitary dysfunction following traumatic brain injury. J. Neurotrauma 21, 685-696.

5. Aimaretti, G., Ambrosio, M.R., Di Somma, C., Gasperi, M., Cannavò, S., Scaroni, C., Fusco, A., Del Monte, P., De Menis, E., Faustini-Fustini, M., Grimaldi, F., Logoluso, F., Razzore, P., Rovere, S., Benvenga, S., degli Uberti, E.C., De Marinis, L., Lombardi, G., Mantero, F., Martino, E., Giordano, G., and Ghigo, E. (2005). Residual pituitary function after brain injury-induced hypopituitarism: a prospective 12-month study. J. Clin. Endocrinol. Metab. 90, 6085-6092.

6. Agha, A., Rogers, B., Sherlock, M., O'Kelly, P., Tormey, W., Phillips, J., and Thompson, C.J. (2004). Anterior pituitary dysfunction in suvivors of traumatic brain injury. J. Clin. Endocrinol. Metab. 89, 4929-4936.

7. Popvic, V., Pekic, S., Pavlovic, D., Maric, N., Jasovic-Gasic, M., Djurovic, B., Medic Stojanoska, M., Zivkovic, V., Stojanovic, M., Doknic, M., Milic, N., Djurovic, M., Dieguez, C., and Casanueva, F.F. (2004). Hypopituitarism as a consequence of traumatic brain injury (TBI) and its possible relation wtih cognitive disabilities and mental distress. J. Endocrinol. Invest. 27, 1048-1054

8. Leal-Cerro, A., Flores, J.M., Rincon, M., Murillo, F., Pujol, M., GarciaPesquera, F., Dieguez, C., and Casanueva, F.F. (2005). Prevalence of hypopituitarism and growth hormone deficiency in adults long-term after severe trauamtic brain injury. Clin. Endocrinol. 62, 525-532.

9. Schneider, H.J., Schneider, M., Saller, B., Petersenn, S., Uhr, M., Husemann, B., von Rosen, F., and Stalla, G.K. (2006). Prevalence of anterior pituitary insuffiency 3 and 12 months after traumatic brain injury. Eur. J. Endocrinol. 154, 259-265.

10. Tanriverdi, F., Senyurek, H., Unluhizarci, K., Selcuklu, A., Casanueva, F.F., and Kelestimur, F. (2006). High risk of hypopituarism after traumatic brain injury: a prospective investigation of anterior pituitary function in the acute phase and 12 months after trauma. J. Clin. Endocrinol. Metab. 91, 2105-2111.

11. Hermann, B.I., Rehder, J., Kahlke, S., Wiedemayer, H., Doerfler, A., Ischebeck, W., Laumer, R., Forsting, M., Stolke, D., and Mann, K. (2006). Hypopituitarism following severe traumatic brain injury. Exp. Clin. Endocrinol. Diabetes 114, 316-321.

12. Klose, M., Juul, A., Poulsgaard, L., Kosteljanetz, M., Brennum, J., and Feldt-Rasmussen, U. (2007). Prevalence and predictive factors of post-traumatic hypopituitarism. Clin. Endocrinol. 67, 193-201.

13. Kelly, D.F., Gonzalo, I.T., Cohan, P., Berman, N., Swerdloff, R., and Wang, C. (2000). Hypopituitarism following traumatic brain injury and aneurysmal subarchnoid hemorrhage: a preliminary report. J. Neurosurg. 93, 743-752.

14. Lieberman, S.A., Oberoi, A.L., Gilkison, C.R., Masel, B.E., and Urban, R.J. (2001). Prevalence of neuroendocrine dysfunction in patients recovering from traumatic brain injury. J. Clin. Endocrinol. Metab. 86, 2752-2756.
15. Ghigo, E., Masel, B., Aimaretti, G., Leon-Carrion, J., Casanueva, F.F., Dominguez-Morales, M.R., Elovic, E., Perrone, K., Stalla, G., Thompson, C., and Urban, R. (2005). Consensus guidelines on screening for hypopituitarism following traumatic brain injury. Brain Inj. 19, 711-724.

16. Aberg, D. (2010). Role of the growth hormone/insulin-like growth factor 1 axis in neurogenesis. Endocr. Dev. 17, 63-76.

17. Arce, V.M., Devesa, P., and Devesa, J. (2013). Role of growth hormone $(\mathrm{GH})$ in the treatment of neural diseases: from neuroprotection to neural repair. Neurosci. Res. 76, 179-186.

18. Freda, P.U. (2003). Pitfalls in the biochemical assessment of acromegaly. Pituitary $6,135-140$.

19. Freda, P.U., Post, K.D., Powell, J.S., and Wardlaw, S.L. (1998). Evaluation of disease status with sensitive measures of growth hormone secretion in 60 postoperative patients with acromegaly. J. Clin. Endocrinol. Metab. 83, 3808-3816.

20. Ho, K.K; 2007 GH Deficiency Consensus Workshop Participants. (2007). Consensus guidelines for the diagnosis and treatment of adults with GH deficiency II: A statement of the GH Research Society in association with the European Society for Pediatric Endocrinology, Lawson Wilkins Society, European Society of Endocrinology, Japan Endocrine Society, and Endocrine Society of Australia. Eur. J. Endocrinol. 157, 695-700.

21. Devesa, J., Devesa, P., and Reimunde, P. (2010). [Growth hormone revisited.] (Spa) Med. Clin. (Barc) 135, 665-670.

22. Lissett, C.A., Jonsson, P., Monson, J.P., and Shalet, S.M.; KIMS International Board. (2003). Determinants of IGF-1 status in a large cohort of growth hormone deficient (GHD) subjects: the role of timing of onset of GHD. Clin. Endocrinol. 59, 773-778.

23. Rosario, E.R., Aqeel, R., Brown, M.A., Sanchez, G., Moore, C., and Patterson, D. (2013). Hypothalamic-pituitary dysfunction following traumatic brain injury affects functional improvement during acute inpatient rehabilitation. J. Head Trauma Rehabil. 28, 390-396.

24. Zgaljardic, D.J., Guttikonda, S., Grady, J.J., Gilkison, C.R., Mossberg, K.A., High, W.M. Jr, Masel, B.E., and Urban, R.J. (2011). Serum IGF1 concentrations in a sample of patients with traumatic brain injury as a diagnostic marker of growth hormone secretory response to glucagon stimulation testing. Clin. Endocrinol. 74, 365-369.

25. Cansanuev, F.F., Castro, A.I., Micic, D., Kelestimur, F., and Dieguez, C. (2009). New guidelines for the diagnosis of growth hormone deficiency in adults. Horm. Res. 71, Suppl 1, 112-115.

26. Biller, B.M., Samuels, M.H., Zagar, A., Cook DM, Arafah, B.M., Bonert, V., Stavrou, S., Kleinberg, D.L., Chipman, J.J., and Hartman, M.L. (2002). Sensitivity and specificity of six tests for the diagnosis of adult GH deficiency. J. Clin. Endocrinol. Metab. 87, 2067-2079.

27. Jones, S.L., Trainer, P.J., Perry, L., Wass, J.A., Bessser, G.M., and Grossman, A. (1994). An audit of the insulin tolerance test in adult subjects in an a acute investigation unit over one year. Clin. Endocrinol. 41, $123-128$.

28. Yuenh, K.C., Biller, B.M., Molitch, M.E., and Cook, D.M,. (2009). Clinical review: is lack of recombinant growth hormone (GH)releasing hormone in the United States a setback or time to consider glucagon testing for adult GH deficiency? J. Clin. Endocrinol. Metab. 94, 2702-2707.

29. Conceicao, F.L., da Costs e Silva, A., Leal Costa, A.J., and Vaisman, M. (2003). Glucagon stimulation test for the diagnosis of GH deficiency in adults. J. Endocrinol. Invest. 26, 1065-1070.

30. Gomez, J.M., Espadero, R.M., Esobar-Jiminez, F., Hawkins, F., Picó, A., Herrera-Pombo, J.L., Vilardell, E., Durán, A., Mesa, J., and Faure, E. (2002). Growth hormone release after glucagon is a reliable test of growth hormone assessment in adults. Clin. Endocrinol. 56, 329-334.

31. Gabellieri, E., Chiovato, L., Lage, M., Castro, A.I., and Casanueva, F.F. (2010). Testing growth hormone deficiency in adults. Front. Horm. Res. 38, 139-144.

32. Rappaport, M., Hall, K.M., Hopkins, K., Belleza, T., and Cope, D.N. (1982). Disability rating scale for severe head trauma: Coma to community. Arch. Phys. Med. Rehabil. 63, 118-123.

33. Ashley, M.J., Persel, C., and Clark, M.C. (2001). Validation of an independent living scale for post-acute rehabilitation applications. Brain Inj. 15, 435-442.

34. Malec, J.F., Moessner, A.M., Kragness, M., and Lezak, M.D. (2000). Refining a measure of brain injury sequelae to predict postacute rehabilitation outcome: rating scale analysis of the Mayo-Portland Adaptability Inventory. J. Head Trauma Rehabil. 15, 670-682. 
35. Willer, B., Ottenbacher, K.J., and Coad, M.L. (1994). The community integration questionnaire: a comparative examination. Am. J. Phys. Med. Rehabil. 73, 103-111.

36. Ashley, M.J., Persel, C.S., Clark, M.C., and Krych, D.K. (1997) Long-term follow-up of post-acute traumatic brain injury rehabilitation: a statistical analysis to test for stability and predictability of outcome. Brain Inj. 11, 677-690.

37. Beck, A.T., Steer, R.A., and Brown G.K. (1996). Beck Depression Inventory-II (BDI-II). The Psychological Corporation: San Antonio,

38. Agha, A., Phillips, J., O'Kelly, P., Tormey, W., and Thompson, C.J. (2005). The natural history of post-traumatic hypopituitarism: implications for assessment and treatment. Am. J. Med. 118, 1416.

39. Bavisetty, S., Bavisetty, S., McArthur, D.L., Dusick, J.R., Wang, C., Cohan, C., Boscardin, W.J., Swerdloff, R., Levin, H., Change, D.J., Muizelaar, J.P., and Kelly, D.F. (2008). Chronic hypopituitarism after traumatic brain injury: risk assessment and relationship to outcome. Neurosurgery 62, 1080-1094.

40. Carlson, N.E., Brenner, L.A., Wierman, M.E., Harrison-Felix, C. Morey, C., Gallagher, S., and Ripley, D. (2009). Hypogonadism on admission to acute rehabilitation is correlated with lower functional status at admission and discharge. Brain Inj. 23, 336-344.

41. Young, T.P., Hoaglin H.M., and Burke, D.T. (2007). The role of serum testosterone and TBI in the in-patient rehabilitation setting. Brain Inj. 21, 645-649.

42. Rothman, M.S., Arciniegas, D.B., Filley, C.M., and Wierman, M.E. (2007). The neuroendocrine effects of traumatic brain injury. J. Neuropsychiatry Clin. Neurosci. 19, 363-372.

43. Bonadanelli, M., Ambrosio, M., Zatelli, M., De Marinis, L., and degli Uberti, E. (2005). Hypopituitarism after traumatic brain injury. Eur. J. Endocrinol. 152, 679-691.

44. Leon-Carrion, J., Leal-Cerro, A., Cabezas, F.M., Atutxa, A.M., Gomez S.G., Cordero, J.M., Moreno, A.S., Ferrari, M.D., and DominguezMorales, M.R. (2007). Cognitive deterioration due to GH deficiency in patients with traumatic brain injury: a preliminary report. Brain Inj. 21, 871-875.

45. High, W.M. Jr., Briones-Galang, M., Clark, J., Gilkison, C., Mossberg, K.A., Zgaljardic, D.J., Masel, B.E., and Urban, R.J. (2010). Effect of growth hormone replacement therapy on cognition after traumatic brain injury. J. Neurotrauma 27, 1565-1575.

46. Carroll, P.V., Christ, E.R., Bengston, B.A., Carlsson, L., Christiansen, J.S., Clemmons, D., Hintz, R., Ho, K., Laron, Z., Sizonenko, P., Sönksen, P.H., Tanaka, T., and Thorne, M. (1998). Growth hormone deficiency in adulthood and the effects of growth hormone replacement: a review. J. Clin. Endocrinol. Metab. 83, 382-395.

47. McGauley, G.A., Cuneo, R.C., Salomon, F., and Sonksen, P.H. (1990). Psychological well-being before and after growth hormone treatment in adults with growth hormone deficiency. Horm. Res. 33, Suppl 4, 52-54.

48. Wang, C., Cunningham, G., Dobs, A., Iranmanesh, A., Matsumoto, A.M., Snyder, P.J., Weber, T., Berman, N., Hull, L., and Swerdloff, R.S. (2004). Long-term testosterone gel (AndroGel) treatment maintains beneficial effects on sexual function and mood, lean and fat mass, and bone mineral density in hypogonadal men. J. Clin. Endocrinol. Metab. 89, 2085-2098.

49. Masel, B.E., and DeWitt, D.S. (2010). Traumatic brain injury: a disease process, not an event. J. Neurotrauma 27, 1529-1540.

Address correspondence to:

Lisa A. Kreber, PhD

Centre for Neuro Skills 5215 Ashe Road

Bakersfield, CA 93313

E-mail:1kreber@neuroskills.com 